\title{
Modified mRNA as a new therapeutic option for pediatric respiratory diseases and hemoglobinopathies
}

\author{
Justin S. Antony, Alexander Dewerth, Ashiqul Haque, Rupert Handgretinger and Michael S.D. Kormann*
}

\begin{abstract}
Background: The immunogenicity and limited stability of conventional messenger RNA (mRNA) has traditionally restricted its potential therapeutic use. In 1992, the first clinical application of mRNA was reported as a potential protein-replacement therapy; however, subsequent investigations have not been made for almost two decades. Recent developments, including increased stability, controlling immunogenicity, as well as utilization of mRNA encoding zinc-finger nucleases (ZFNs), transcription activator-like effector nucleases (TALENs), and CRISPR-Cas9, have implicated modified mRNA as a very promising option for cancer immunotherapy, vaccines, protein expression replacement, and genome editing. This review aims to offer a summary of our present understanding of and improvements in mRNA-based drug technologies, along with a focus on the role in therapeutic options for pediatric respiratory diseases and hemoglobinopathies.
\end{abstract}

Conclusions: This mini review summarizes the recent advances in modified mRNA-based therapy and its potential therapeutic effect in treating major pediatric diseases.

Keywords: Modified mRNA, Pediatric diseases, Gene therapy

\section{Findings}

Introduction

Pediatric respiratory illness is a major cause of mortality and morbidity among infants and young children as they are more susceptible to respiratory diseases [1]. Asthma, tuberculosis, bronchiectasis, and bronchopulmonary dysplasia are chronic respiratory diseases in children. In addition, congenital respiratory disorders such as cystic fibrosis and primary ciliary dyskinesia are observed at lower incidence in children [2,3]. Next to the lung diseases, hemoglobinopathies are the most common genetic disorders in pediatrics as at least 60,000 severely affected children are born every year $[4,5]$. Although treatments are available for both clinical pictures, the major issue appears to be its limited effectiveness, thus providing only a short-term cure $[5,6]$. However, recent advancements in science have paved the way to treat such diseases at the molecular level as new therapeutic targets

\footnotetext{
* Correspondence: michael.kormann@med.uni-tuebingen.de

Department of Pediatrics I-Pediatric Infectiology and Immunology,

Translational Genomics and Gene Therapy in Pediatrics, University of Tübingen, Tübingen, Germany
}

and pathways are uncovered. In this review, we focus on common pediatric respiratory illness and hemoglobinopathies that are potentially amenable to gene therapy.

For two decades, gene therapy has been focused on plasmid DNA and viral DNA, with severe consequences in early key studies [7]. However, gene therapy has greatly evolved and has become a major focus in nucleotidebased gene therapy. Karikó et al. and Kormann et al. demonstrated that chemical modification of messenger RNA (mRNA) resulted in mRNA transcripts being less immunogenic and more efficiently translated in vitro [8] and in vivo [9]. Such chemically modified messenger RNA (mod. mRNA) has many advantages compared to other therapeutic nucleic acids. The most important features comprise a transient protein expression, reduced immunogenicity, superior translation efficiency, and pharmaceutical safety, as mod. mRNA does not integrate into the host genome $[8,10,11]$. Current progress in targeted genome editing mediated by nucleases and encoded by mod. mRNA to express engineered nucleases such as the CRISPR-Cas9 system, zinc-finger nucleases (ZFNs), and TAL effector nucleases (TALENs) will undoubtedly 
translate basic research findings to novel molecular therapeutics in the treatment of pediatric diseases $[12,13]$. The principle and applications of mod. mRNA are illustrated in Fig. 1.

\section{Mod. mRNA: a better tool for gene therapy?}

In a landmark publication, Warren et al. implicated chemically modified mRNA as a powerful tool to express proteins of interest in target cells in vitro. These authors showed that repeated administration of mod. mRNA encoding the Yamanaka factors KLF4, c-MYC, OCT4, and SOX2 could reprogram terminally differentiated human fibroblasts into pluripotent myofibroblasts without the need for retroviral vectors [14]. Consequently, the study provided an efficient way to reprogram cell fate without the risk of potential genomic integration. Subsequent studies have extended these initial observations of mod. mRNA-encoded protein expression to in vivo models, demonstrating specific expression in organs such as the heart, skeletal muscle, and lung $[9,15,16]$.

\section{Mod. mRNA: reduced immunogenicity and increased stability}

To achieve a beneficial therapeutic effect, reduced activation of innate immune receptors and an increased biological stability are key issues that need to be addressed when considering future clinical applications of mod. mRNA. Chemical substitutions such as the replacement of uridine by pseudouridine $(\Psi)$ or cytosine by 5 -methylcytosine $(\mathrm{m} 5 \mathrm{C})$ on target mRNA ultimately allow them to evade innate immune responses such as Toll-like receptor (TLRs) signaling pathways [8]. A study by Kormann et al. demonstrated that combining chemical modifications, such as the replacement of $25 \%$ of uridine and cytidine with 2-thiouridine ( $\mathrm{s} 2 \mathrm{U}$ ) and $\mathrm{m} 5 \mathrm{C}$ reduced recognition of target mRNA through pattern recognition receptors, including TLR3, TLR7, TLR8, and RIG-I in human PBMCs.

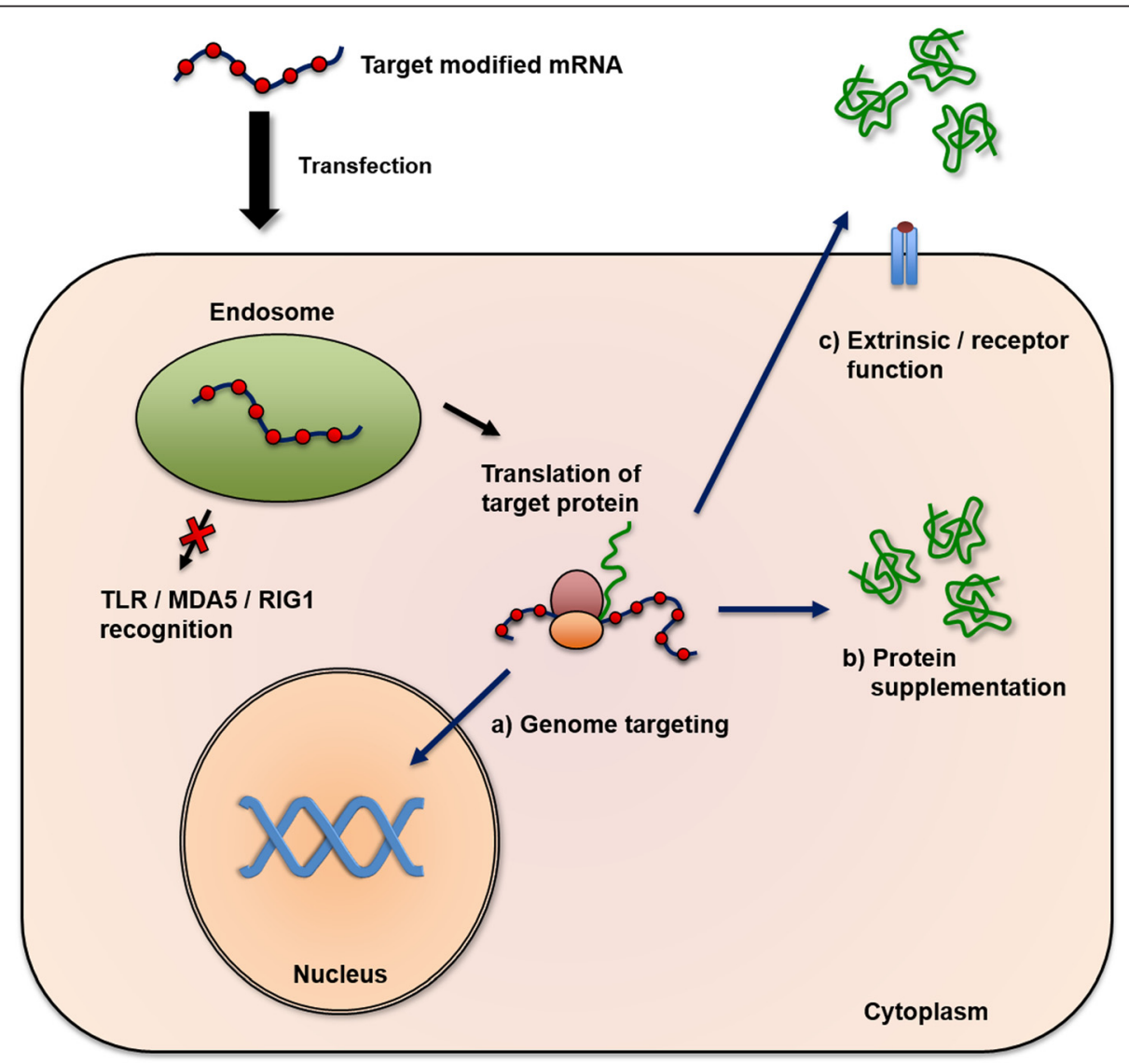

Fig. 1 The principle and applications of modified mRNA. Modified mRNA can be transfected via several routes to the target cell, e.g., intraperitoneally, intravenously, or intratracheally. After endocytosis, the incorporation of naturally occurring, noncanonical nucleosides (indicated as red dots) into in vitro transcribed mRNA prevents activation of and consequently degradation by endosomal (Toll-like receptors, TLRs) and cytoplasmatic (MDA5, RIG1) mRNASensors, thus being efficiently translated into a functional protein at the cell's ribosomes. Different target applications can be applied for modified mRNA: (a) genome targeting, resulting in functional nucleases (e.g., zink-finger nucleases, CRISPR/Cas9) that bind and create sequence-specific double-strand breaks within the genome to facilitate gene correction, gene replacement or gene knock-out; (b) protein supplementation, where endogeneous protein malfunction can be overcome by restoring normal protein function; $(c)$ extrinsic/receptor function in case of improper cell signaling 
In addition, the same modifications also facilitated in vivo delivery of the respective mRNA [9]. These findings were verified by a subsequent study and confirmed that chemical modification effects in reduced innate immune recognition [17]. Very recently, it has been shown that the incorporation of $\mathrm{N}^{1}$-methylpseudouridine $(\mathrm{m} 1 \Psi)$ in mRNA resulted in innate immune evasion and increased translational capacity in vitro and in vivo [18]. Next to chemical modifications, other approaches to enhance stability and translational efficacy of mRNA include the use of phosphorothioate $5^{\prime}$ cap analogs, HPLC purification, and polyadenylation tail by a defined number of adenosines as well as the incorporation of stabilizer proteins [19-22]. The progress in lipid nanoparticle mediated delivery of mod. mRNA into target cells in vivo by various routes provided an efficient translation [23].

\section{Nuclease-encoding mRNA: a novel strategy for permanent cure} Due to the transient expression of mod. mRNA, its use will be difficult in the treatment of chronic genetic diseases, as they require longer-term solutions. To circumvent this, nuclease-encoded chemically mod. mRNA (nec-mRNA) has been described as a novel delivery paradigm. Expression of mRNA encoding zinc-finger nucleases (ZFNs) or transcription activator-like effector nucleases (TALENs) resulted in successful genome editing and subsequently the correction of surfactant protein B (SP-B) deficiency in mice [13]. The principle of nec-mRNA is illustrated in Fig. 2. Due to the rapid yet transient burst of expression of necmRNA, off-target effects will be minimized when compared to long-term expression by viral or plasmid vectors. In addition, the expression kinetics can be fine-tuned by different combinations of chemical modifications [24]. Thus, the proposed strategy might provide permanent gene correction by targeting stem cells. Moreover, the use of single-stranded oligodeoxynucleotides (ssODNs)-short, single-stranded DNA-based repair templates-for gene targeting of short nuclear polymorphisms (SNPs) would completely abrogate the need for viral vectors, resulting in patient-specific gene correction and thus an ultimate cure.

\section{Proof of principle: use of mod. mRNA to treat pediatric diseases}

Cystic fibrosis, SP-B deficiency, asthma, $\beta$ thalassemia, and sickle cell anemia are all genetic diseases that can affect children. With its potential for protein replacement and gene correction, mod. mRNA presents a powerful tool to minimize or even cure these conditions. The next part of the review will focus on the present evidence of positive results using mod. mRNA against genetic disease.

\section{$S P-B$ deficiency}

Surfactant protein B (SP-B) is a pulmonary surfactant protein that reduces surface tension and prevents the collapse of alveoli. Congenital SP-B deficiency is a rare, lethal, and monogenic disease induced mostly by lossof-function mutations on both SFTPB alleles. The administration of surfactants, corticosteroids, and other immunosuppressants, repeated lung lavage, and ultimately lung transplantation are the only therapeutic interventions currently available. However, these treatments show poor efficacy and outcome [25]. In mice, repeated administration of mod. SP-B mRNA by intratracheal high-pressure spraying significantly increased SP-B protein levels, resulting in the elimination of pulmonary inflammation and a significant increase in survival [9]. Furthermore, a single intratracheal application of nec-mRNA combined with AAV-encoded repair template increased the life span of SP-B deficient mice due to genetic correction in alveolar type II cells [13].

\section{Cystic fibrosis}

Cystic fibrosis (CF) is the most life-limiting monogenic disease in Caucasian populations and is caused by a mutation in the gene that encodes the cystic fibrosis transmembrane and conductance regulator (CFTR). CF is characterized by altered epithelial mucus secretion in several organs, most severely in the pulmonary epithelium [26]. In spite of the various clinical trials performed with viral and nonviral vector-mediated gene therapy approaches in CF patients, these techniques have shown low efficacy [27]. Therefore, a viable alternative therapy has yet to be developed, e.g., via repeated administration of therapeutic nucleic acids that penetrate the mucus of CF patients. Modified mRNA has already shown great potential to increase protein expression in the lungs [9]. It is estimated that only $10 \%$ of normal levels of CFTR activity is sufficient to avoid the disintegrating effects seen in CF [28] making mod. mRNA a promising tool to restore adequate CFTR expression in the lungs. In addition, the transfection of chemically modified CFTR mRNA in mutated CFBE41o- cells restored cAMP-induced CFTR currents similar to wild type cells due to the mRNA-driven replacement of functionally active channels [20].

\section{Pediatric asthma}

Asthma is the most common chronic inflammatory disorder in childhood and is associated with airway hyper-responsiveness leading to recurrent episodes of wheezing, breathlessness, chest tightness, coughing, and airflow obstruction. However, in children below 5 years of age, clinical symptoms of asthma differ individually in a nonspecific manner. Usually, inhalation of corticosteroids and bronchodilators is the first choice when it comes to the treatment of asthma. However, some patients face the clinical picture of corticosteroid resistance as a consequence to repeated corticosteroid administration. In this case, treatment by means of gene replacement could be an 


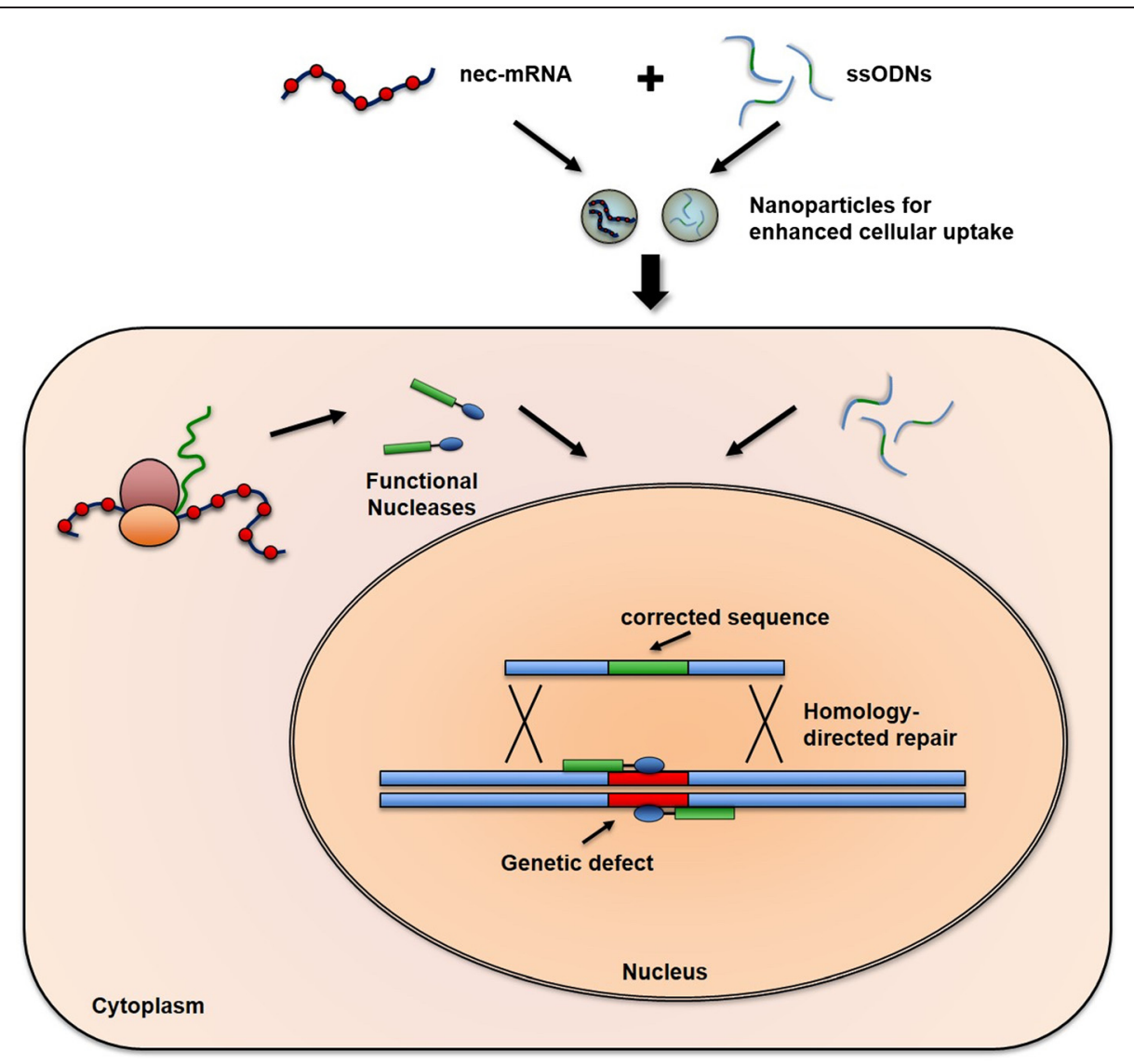

Fig. 2 SNP correction using nec-mNRA and ssODNs. To facilitate site-specific gene correction of single nucleotide polymorphisms (SNPs), nec-mRNA and single-stranded oligodeoxynucleotides (ssODNs) can be efficiently administered to target cells (e.g., lung cells) by complexing both into positively charged nanoparticles for enhanced cellular uptake. Once in the cell, nec-mRNA gets translated into a functional nuclease that subsequently translocates to the nucleus and binds at sequence-specific sites next to the SNP. Generating a double-strand break (DSB), the genomic mutation can be corrected in the presence of SsODNs by means of homology-directed repair, resulting in proper gene function

alternative route and possibly benefit these patients. One such target for gene therapy would be to influence Th2 cytokine reactions [29] by inducing regulatory $\mathrm{T}$ cells. Mays et al. demonstrated that administration of modified Foxp3 mRNA in a time- and site-specific fashion in murine lungs prevented allergic asthma in vivo, while suppressing Th2 responses [15].

\section{$\beta$-Thalassemia and sickle cell disease}

As one of the most common single-gene defects across the globe, $\beta$-thalassemia represents a significant burden for affected patients and families. Driven by various mutations in the $\beta$-globin gene cluster, the disease is characterized by a reduction or absence of $\beta$-globin gene expression. Ultimately, this results in a lack of functional adult hemoglobin ( $\mathrm{HbA}$ ), which is composed of two alpha- and two beta-globin chains $\left(\alpha_{2} \beta_{2}\right)$ [4]. Without sufficient hemoglobin, red blood cells develop abnormally, leading to severe anemia. This imbalance in $\alpha$ - and $\beta$-globin production also hinders erythroid precursor maturation, resulting in ineffective erythropoiesis.

Mutations in the human hemoglobin beta $(H B B)$ gene cause $\beta$-thalassemia and those that are homozygous for a given mutation suffer severe anemia. In children, anemia begins to develop within the first month of life and infants fail to thrive. Currently, allogeneic bone marrow transplantation and hematopoietic stem cell transfusion are the only available curative schemes; in turn, these treatments are limited to a minority of patients due to the availability of histocompatible donors. However, gene therapy based on autologous transplantation of genetically corrected hematopoietic stem cells (HSCs) shows high potential as a cure, since it is not restricted to histocompatible donors and immunosuppression. The lentiviral delivery of a normal HBB gene into hematopoietic stem cells could be shown to result in therapeutic benefit [30]. However, viral vectors always possess the risk of causing insertional mutagenesis. Recent developments in gene correction using nec-mRNA encoding proteins such as ZFNs, 
TALENS, and CRISPR-Cas9 have shown high potential to effectively correct genes while mitigating the danger of internal mutagenesis. It has been reported that using CRISPR-Cas9 (with modified mRNA) to cleave the HBB gene and piggyBac for homologous recombination selection can correct two different $\beta$-thalassemia mutations and thus reduce the genetic status to a heterozygous state, where patients are only mildly anemic and capable of leading normal lives [31].

Sickle cell disease is caused by mutations in the protein-coding $H B B$ gene. Replacement of A to $\mathrm{T}$ results in valine instead of glutamic acid. Both copies of the gene need to contain this particular mutation to cause anemia. A recent report suggests that seamless $H B B$ gene correction is possible using TALENs and piggyBac [32]. Together with its precise correcting efficiency and high modularity, these nucleases represent a promising tool to correct such a SNP [33].

\section{Future directions}

mRNA has tremendous potential for both gene therapy and gene correction approaches. However, as with every new technology, a number of methodological improvements and specific challenges need to be addressed. The chemical modification needs to be adapted to the target cell type and to the transfection reagent used. In addition, the incorporation of chemically modified nucleosides into mRNA increases protein stability but decreases translational efficiency [24]. Thus, it is recommended that mRNA formulations undergo extensive optimization to achieve a therapeutic benefit. Furthermore, in order to achieve permanent cures for genetic diseases as that mentioned above, the nec-mRNA and repair templates must be directed to stem/progenitor cells by targeted cell therapy.

In conclusion, the therapeutic potential of mod. mRNA has been rapidly recognized for the treatment of pediatric diseases. However, further improvements in systemic delivery, consideration of different cell types or different organs, and effective chemical modifications for each such type are pivotal for efficient utilization. Combining efforts to overcome cell turnover together with appropriate animal models will enable the field to make continued progress and to reach the step into the clinic.

\section{Competing interests}

M.S.D.K. is listed as main inventor on a patent application related to modified mRNA. M.S.D.K. is an inventor on a patent licensed to the biopharmaceutical company, Ethris $\mathrm{GmbH}$.

\section{Authors' contributions}

J.S.A. wrote the main part of the manuscript, followed by valuable contributions of A.D., who created the figures and gave input to the manuscript. A.H. and R.H. contributed equally to the concept and performed thorough proofreading. M.S.D.K. drafted the final version of the manuscript. All authors read and approved the final manuscript.

\section{Acknowledgements}

We are grateful to D. Kormann for valuable input in the structure of the graphics and to D. Hartl for his help to create this paper from a clinical view that for sure will be interesting for researchers as well as clinicians working in the field to maybe set different future prospects.

Received: 24 March 2015 Accepted: 16 November 2015

Published online: 20 November 2015

\section{References}

1. Gibson GJ, Loddenkemper R, Lundback B, Sibille Y (2013) Respiratory health and disease in Europe: the new European Lung White Book. Eur Respir J 42(3):559-563. doi:10.1183/09031936.00105513

2. Zar HJ, Ferkol TW (2014) The global burden of respiratory disease-impact on child health. Pediatr Pulmonol 49(5):430-434. doi:10.1002/ppul.23030

3. Zar HJ, Mulholland K (2003) Global burden of pediatric respiratory illness and the implications for management and prevention. Pediatr Pulmonol 36(6):457-461. doi:10.1002/ppul.10345

4. Higgs DR, Engel JD, Stamatoyannopoulos G (2012) Thalassaemia. Lancet 379(9813):373-383. doi:10.1016/S0140-6736(11)60283-3

5. Ngo DA, Steinberg MH (2015) Genomic approaches to identifying targets for treating beta hemoglobinopathies. BMC Med Genomics 8(1):44. doi:10.1186/s12920-015-0120-2

6. Kumar S, Tana A, Shankar A (2014) Cystic fibrosis - what are the prospects for a cure? Eur J Intern Med 25(9):803-807. doi:10.1016/j.ejim.2014.09.018

7. Anson DS (2004) The use of retroviral vectors for gene therapy - what are the risks? A review of retroviral pathogenesis and its relevance to retroviral vector-mediated gene delivery. Genet Vaccines Ther 2(1):9. doi:10.1186/ 1479-0556-2-9

8. Kariko K, Muramatsu H, Welsh FA, Ludwig J, Kato H, Akira S, Weissman D (2008) Incorporation of pseudouridine into mRNA yields superior nonimmunogenic vector with increased translational capacity and biological stability. Mol Ther 16(11):1833-1840. doi:10.1038/mt.2008.200

9. Kormann MS, Hasenpusch G, Aneja MK, Nica G, Flemmer AW, Herber-Jonat S, Huppmann M, Mays LE, Illenyi M, Schams A, Griese M, Bittmann I, Handgretinger R, Hartl D, Rosenecker J, Rudolph C (2011) Expression of therapeutic proteins after delivery of chemically modified mRNA in mice. Nat Biotechnol 29(2):154-157. doi:10.1038/nbt.1733

10. Kuhn AN, Beibetaert T, Simon P, Vallazza B, Buck J, Davies BP, Tureci O, Sahin $U$ (2012) mRNA as a versatile tool for exogenous protein expression. Curr Gene Ther 12(5):347-361

11. Kormann M, Yamamoto A, Rosenecker J, Rudolph C (2009) Current prospects for mRNA gene delivery. Eur J Pharm Biopharm 71(3):484-489. doi:10.1016/j.ejpb.2008.09.016

12. Sander JD, Joung JK (2014) CRISPR-Cas systems for editing, regulating and targeting genomes. Nat Biotechnol 32(4):347-355. doi:10.1038/nbt.2842

13. Dewerth A, Mahiny AJ, Mays LE, Alkhaled M, Mothes B, Malaeksefat E, Loretz B, Rottenberger J, Brosch DM, Reautschnig P, Surapolchai P, Zeyer F, Schams A, Carevic M, Bakele M, Griese M, Schwab M, Nurnberg B, Beer-Hammer S, Handgretinger R, Hartl D, Lehr CM, Kormann MS (2015) In vivo genome editing using nuclease-encoding mRNA corrects SP-B deficiency. Nat Biotechnol 33(6):584-586. doi:10.1038/nbt.3241

14. Warren L, Manos PD, Ahfeldt T, Loh YH, Li H, Lau F, Ebina W, Mandal PK, Smith ZD, Meissner A, Daley GQ, Brack AS, Collins JJ, Cowan C, Schlaeger TM, Rossi DJ (2010) Highly efficient reprogramming to pluripotency and directed differentiation of human cells with synthetic modified mRNA. Cell Stem Cell 7(5):618-630. doi:10.1016/j.stem.2010.08.012

15. Mays LE, Ammon-Treiber S, Mothes B, Alkhaled M, Rottenberger J, MullerHermelink ES, Grimm M, Mezger M, Beer-Hammer S, Von Stebut E, Rieber N, Nurnberg B, Schwab M, Handgretinger R, Idzko M, Hartl D, Kormann MS (2013) Modified Foxp3 mRNA protects against asthma through an IL-10dependent mechanism. J Clin Invest 123(3):1216-1228. doi:10.1172/JCl65351

16. Zangi L, Lui KO, Von Gise A, Ma Q, Ebina W, Ptaszek LM, Spater D, Xu H, Tabebordbar M, Gorbatov R, Sena B, Nahrendorf M, Briscoe DM, Li RA, Wagers AJ, Rossi DJ, Pu WT, Chien KR (2013) Modified mRNA directs the fate of heart progenitor cells and induces vascular regeneration after myocardial infarction. Nat Biotechnol 31(10):898-907. doi:10.1038/nbt.2682

17. Andries $O$, De Filette M, De Smedt SC, Demeester J, Van Poucke M, Peelman L, Sanders NN (2013) Innate immune response and programmed cell death following carrier-mediated delivery of unmodified mRNA to respiratory cells. J Control Release 167(2):157-166. doi:10.1016/j.jconrel.2013.01.033 
18. Andries O, Mc Cafferty S, De Smedt SC, Weiss R, Sanders NN, Kitada T (2015) $\mathrm{N}$-methylpseudouridine-incorporated mRNA outperforms pseudouridineincorporated mRNA by providing enhanced protein expression and reduced immunogenicity in mammalian cell lines and mice. J Control Release. doi:10.1016/j.jconrel.2015.08.051

19. Kuhn AN, Diken M, Kreiter S, Selmi A, Kowalska J, Jemielity J, Darzynkiewicz E, Huber C, Tureci O, Sahin U (2010) Phosphorothioate cap analogs increase stability and translational efficiency of RNA vaccines in immature dendritic cells and induce superior immune responses in vivo. Gene Ther 17(8):961-971. doi:10.1038/gt.2010.52

20. Bangel-Ruland N, Tomczak K, Fernandez Fernandez E, Leier G, Leciejewski B, Rudolph C, Rosenecker J, Weber WM (2013) Cystic fibrosis transmembrane conductance regulator-mRNA delivery: a novel alternative for cystic fibrosis gene therapy. J Gene Med 15(11-12):414-426. doi:10.1002/jgm.2748

21. Holtkamp S, Kreiter S, Selmi A, Simon P, Koslowski M, Huber C, Tureci O, Sahin U (2006) Modification of antigen-encoding RNA increases stability, translational efficacy, and T-cell stimulatory capacity of dendritic cells. Blood 108(13):4009-4017. doi:10.1182/blood-2006-04-015024

22. Peng SS, Chen CY, Xu N, Shyu AB (1998) RNA stabilization by the AU-rich element binding protein, HuR, an ELAV protein. EMBO J 17(12):3461-3470. doi:10.1093/emboj/17.12.3461

23. Pardi N, Tuyishime S, Muramatsu H, Kariko K, Mui BL, Tam YK, Madden TD, Hope MJ, Weissman D (2015) Expression kinetics of nucleoside-modified mRNA delivered in lipid nanoparticles to mice by various routes. J Control Release. doi:10.1016/j.jconrel.2015.08.007

24. Uchida S, Kataoka K, Itaka K (2015) Screening of mRNA chemical modification to maximize protein expression with reduced immunogenicity. Pharmaceutics 7(3):137-151. doi:10.3390/pharmaceutics7030137

25. Hamvas A, Nogee LM, Mallory GB Jr, Spray TL, Huddleston CB, August A, Dehner LP, deMello DE, Moxley M, Nelson R, Cole FS, Colten HR (1997) Lung transplantation for treatment of infants with surfactant protein $B$ deficiency. J Pediatr 130(2):231-239

26. Matsui H, Verghese MW, Kesimer M, Schwab UE, Randell SH, Sheehan JK, Grubb BR, Boucher RC (2005) Reduced three-dimensional motility in dehydrated airway mucus prevents neutrophil capture and killing bacteria on airway epithelial surfaces. J Immunol 175(2):1090-1099

27. Prickett M, Jain M (2013) Gene therapy in cystic fibrosis. Transl Res 161(4): 255-264. doi:10.1016/j.trsl.2012.12.001

28. Ratjen F, Doring G (2003) Cystic fibrosis. Lancet 361(9358):681-689. doi:10. 1016/S0140-6736(03)12567-6

29. Lykouras D, Karkoulias K, Tourmousoglou C, Koletsis E, Spiropoulos K, Dougenis D (2013) Gene therapy perspectives against diseases of the respiratory system. Gene Therapy - Tools and Potential Applications. doi:43290

30. Cavazzana-Calvo M, Payen E, Negre O, Wang G, Hehir K, Fusil F, Down J, Denaro M, Brady T, Westerman K, Cavallesco R, Gillet-Legrand B, Caccavelli L, Sgarra R, Maouche-Chretien L, Bernaudin F, Girot R, Dorazio R, Mulder GJ, Polack A, Bank A, Soulier J, Larghero J, Kabbara N, Dalle B, Gourmel B, Socie G, Chretien S, Cartier N, Aubourg P, Fischer A, Cornetta K, Galacteros F, Beuzard Y, Gluckman E, Bushman F, Hacein-Bey-Abina S, Leboulch P (2010) Transfusion independence and HMGA2 activation after gene therapy of human beta-thalassaemia. Nature 467(7313):318-322. doi:10.1038/ nature09328

31. Xie F, Ye L, Chang JC, Beyer Al, Wang J, Muench MO, Kan YW (2014) Seamless gene correction of beta-thalassemia mutations in patient-specific iPSCs using CRISPR/Cas9 and piggyBac. Genome Res 24(9):1526-1533. doi:10.1101/gr.173427.114

32. Sun N, Zhao H (2014) Seamless correction of the sickle cell disease mutation of the HBB gene in human induced pluripotent stem cells using TALENs. Biotechnol Bioeng 111(5):1048-1053. doi:10.1002/bit.25018

33. Joung JK, Sander JD (2013) TALENs: a widely applicable technology for targeted genome editing. Nat Rev Mol Cell Biol 14(1):49-55. doi:10.1038/nrm3486

\section{Submit your manuscript to a SpringerOpen ${ }^{\odot}$ journal and benefit from:}

- Convenient online submission

- Rigorous peer review

- Immediate publication on acceptance

- Open access: articles freely available online

- High visibility within the field

- Retaining the copyright to your article

Submit your next manuscript at $>$ springeropen.com 